\title{
ARTICLE
}

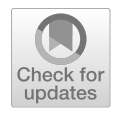

Cite as

Nano-Micro Lett.

(2020) 12:159

Received: 28 April 2020

Accepted: 13 July 2020

Published online: 8 August 2020

(C) The Author(s) 2020

\section{Flexible Waterproof Piezoresistive Pressure Sensors with Wide Linear Working Range Based on Conductive Fabrics}

\author{
Hongcheng $\mathrm{Xu}^{1,2} \bowtie$, Libo Gao ${ }^{1,2}$, Yuejiao Wang ${ }^{3,4}, \mathrm{Ke}^{\mathrm{Cao}}{ }^{1,2}$, Xinkang $\mathrm{Hu}^{1,2}$, \\ Liang Wang ${ }^{5}$, Meng Mu ${ }^{1,2}$, Min Liu ${ }^{1,2}$, Haiyan Zhang ${ }^{1,2}$, Weidong Wang ${ }^{1,2} \bowtie$, \\ Yang $\mathrm{Lu}^{2,3,4} \bowtie$ \\ Hongcheng $\mathrm{Xu}$, Libo Gao, and Yuejiao Wang have contributed equally to this work. \\ $\bowtie$ Hongcheng Xu, xuhongcheng@stu.xidian.edu.cn; Libo Gao, lbgao@xidian.edu.cn; Weidong Wang, \\ wangwd@mail.xidian.edu.cn; Yang Lu, yanglu@cityu.edu.hk \\ 1 School of Mechano-Electronic Engineering, Xidian University, Xi' an 710071, People's Republic of China \\ 2 CityU-Xidian Joint Laboratory of Micro/Nano-Manufacturing, Xi' an 710071, People's Republic of China \\ 3 Department of Mechanical Engineering, City University of Hong Kong, Kowloon 999077, Hong Kong SAR, \\ People's Republic of China \\ 4 Nano-Manufacturing Laboratory (NML), Shenzhen Research Institute of City University of Hong Kong, \\ Shenzhen 518057, People's Republic of China \\ 5 Micro-/Nano-technology Research Center, State Key Laboratory for Manufacturing Systems Engineering, \\ Xi' an Jiaotong University, Xi' an 710049, People's Republic of China
}

\section{HIGHLIGHTS}

- The laser-engraved method was introduced to fabricate the electrode for the sensor.

- The sensor showed a wide linear working range, superior sensitivity, and fast response time and also exhibited excellent viability in a wet situation.

- Wireless integrated network sensors successfully monitored the health states.

\section{ABSTRACT}

Developing flexible sensors with high working performance holds intense interest for diverse applications in leveraging the Internet-of-things (IoT) infrastructures. For flexible piezoresistive sensors, traditionally most efforts are focused on tailoring the sensing materials to enhance the contact resistance variation for improving the sensitivity and working range, and it, however, remains challenging to simultaneously achieve flexible sensor with a linear working range over a high-pressure region (>100 kPa) and keep a reliable sensitivity. Herein, we devised a laser-
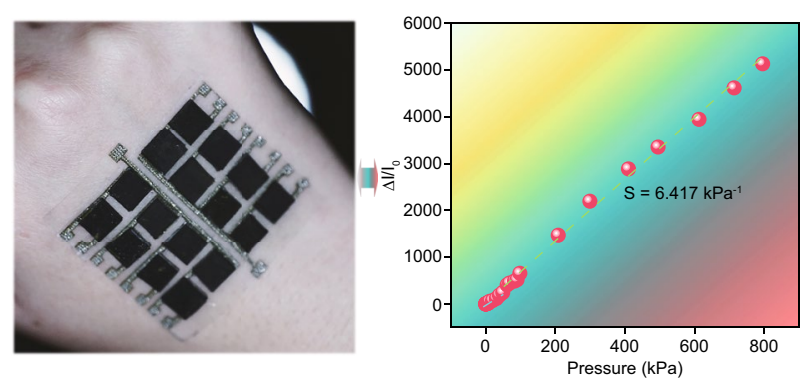
engraved silver-coated fabric as "soft" sensor electrode material to markedly advance the flexible sensor's linear working range to a level of $800 \mathrm{kPa}$ with a high sensitivity of $6.4 \mathrm{kPa}^{-1}$ yet a fast response time of only $4 \mathrm{~ms}$ as well as long-time durability, which was rarely reported before. The integrated sensor successfully routed the wireless signal of pulse rate to the portable smartphone, further demonstrating its potential as a reliable electronic. Along with the rationally building the electrode instead of merely focusing on sensing materials capable of significantly improving the sensor's performance, we expect that this design concept and sensor system could potentially pave the way for developing more advanced wearable electronics in the future.

KEYWORDS Flexible sensor; Piezoresistive; Graphite flakes; Laser engraving; Silver fabrics 


\section{Introduction}

monitoring [5-8], and human-machine interactions (HMI) [9-11]. To expand the feasibility of the piezoresistive sensor for diversified practical applications, they should have a linear pressure-sensing capability within large dynamic sensing ranges to constantly maintain their high sensitivity from lowpressure $(<10 \mathrm{kPa})$ to high-pressure region $(>100 \mathrm{kPa}$, even near $1 \mathrm{MPa}$ ) [12-14]. So far, however, this is still a great challenging task for this kind of pressure sensor [15].

In parallel, although so far extensive new approaches that have been developed to address the balance between the high sensitivity and wide working range, not all are fully effective, and many necessitated tedious strategies for practical application [16]. In principle, the piezoresistive effect mainly derived from the contact resistance changes between the sensing material and the electrode or the bulk resistance within the sensing material [17]. Compared to the latter, the contact resistance contributes to the most conductivity variation under the same applied pressure [18]. The reason is that a small increase in the contact area between the electrode and the active material leads to a significant decrease in the contact resistance. Inspired by this, constructing ordered rough architectures (e.g., the planar, microdome, micropyramid, and the micropillar structure) on the sensing materials were extensively studied to increase the contact variation in improving the sensitivity and working range [12, 19-22]. Fewer reports, however, including our previous studies, achieved a wide working range of over $100 \mathrm{kPa}$ with superior sensitivity were studied through the development of the sensing materials [23-25]. Regarding this, more deep experimental research on the modification of electrode structure instead of merely the sensing materials is needed to attempt to overcome this hurdle and excavate more potential to improve both the working range and sensitivity. As a typical demonstration, our recent work used an insulating bio-derived porous layer as an insulating spacer between the sensing and electrode materials achieved a practical and energy-conservation approach [26].

In this study, the laser-engraved soft silver-coated fabric (SF) with ordered textures was regarded as the sensor's integrated electrode, while the graphite flakes (GFs)-modified non-woven fabrics (G-nWF) were served as sensing materials. Benefited from the unique structure of the electrode and sensing materials' random rough surface, we surprisingly push the flexible sensor's linear working range to a new level of $800 \mathrm{kPa}$ with a high sensitivity of $6.4 \mathrm{kPa}^{-1}$, a fast response time of
Flexible pressure sensor, especially the piezoresistive sensor, is widely employed in electronic skin [1-4], healthcare only $4 \mathrm{~ms}$, and long-time durability. Our sensor device also exhibited improved viability in the wet environment due to its rationally designed microscale polymeric forests. Finally, a wireless signal wearable sensor device was successfully demonstrated to send the pulse rate information to the portable smartphone. The methodology and platform offered a powerful tool for designing and creating advanced flexible sensor with a wide working range and superior sensitivity through the "electrode" aspect and which also could potentially be used for other advanced electronics.

\section{Experimental Section}

\subsection{Preparation of the PDMS Micropillar Film (PDMS MPF)}

Prior to the preparation of the PDMS MPF, the PDMS solution was firstly prepared by mixing a base (Dow Corning, Sylgard $184 \mathrm{~A}$ ) with a curing agent (Dow Corning, Sylgard $184 \mathrm{~B}$ ) with a weight mixing ratio of 10:1. The stainless steel mold with cylinder pores $(200 \mu \mathrm{m}$ in diameter) was initially manufactured carefully by the laser cutting machine. Then, a certain amount of CNT solution (5 mg mL $\mathrm{m}^{-1}$ in isopropyl alcohol (IPA)) was sprayed onto the above stainless steel foil with $1 \mathrm{~mL} \mathrm{~cm}^{-2}$. After the CNT solution was fully evaporated at $70{ }^{\circ} \mathrm{C}$ for $30 \mathrm{~min}$, the prepared PDMS solution was poured into the mold and together put into the vacuum-drying oven to sufficiently remove the gas bubbles with the subsequence of heating at $70^{\circ} \mathrm{C}$ for $1 \mathrm{~h}$. Finally, the MPF covered with CNT was carefully peeled off from the substrate.

\subsection{Assembly of the Flexible Sensor}

The flexible sensor mainly composed of the laser-carved integrated electrode and sensing materials. The SF (purchased from Dongguan Fenda Optoelectronics Co. LTD) was firstly mounted onto the PI/PET film $(100 \mu \mathrm{m}$ in thickness) tightly. And then, a laser etching system (maximum power of $7 \mathrm{~W}$ ) with a wavelength of $405 \mathrm{~nm}$ was employed to etching the unused part, followed by using a tweezer to remove the residual SF to assist the formation of the 
designed soft electrode panel. The laser speed and power were set to be $1500 \mathrm{~mm} \mathrm{~s}^{-1}$ and $28 \%$, respectively. The spacing distance both was set to be $800 \mu \mathrm{m}$, which was the optimal size can be reached for this patterning method. For the preparation of the sensing materials, the non-woven fabrics were sequentially treated with alcohol and deionized (DI) water with the following of drying the substrate at ambient temperature. And then, the prepared sample was made conductive by directly immersing into the graphene solution $\left(2 \mathrm{mg} \mathrm{mL}^{-1}\right)$ according to our previous research [26]. After assembling the PDMS MPF, G-nWF, and flexible SF electrode together, the self-cleaning flexible sensor was successfully fabricated.

\subsection{Phase and Structural Characterization}

The sample morphology was observed via a scanning electron microscope (SEM, FEI-Quanta ${ }^{\mathrm{TM}} 450$ FEG). The composition and crystalline structure were analyzed on transmission electron microscopy (TEM, JEOLJEM-2100F).

\subsection{Micro-mechanical Testing of GFs}

The micro-mechanical tests were conducted inside a JEOL 2100 TEM using a Nanofactory TEM-scanning tunneling microscopy (TEM-STM) holder. GFs were glued to a 200mesh copper half-grid and then glued to a gold rod using silver-conductive epoxy (Ted pella, inc) on the one side of the holder. On the other side, a tungsten needle was inserted in the holder for in situ manipulation of individual GFs.

\subsection{Electro-mechanical Measurement of the Sensor}

Compression or tensile tests were applied by a universal mechanical testing machine (Zhi Qu, ZQ-990B). Corresponding resistance or current change of the sensor was directly recorded by the source meter (Keithley 2400) and electrochemical workstation (Chenhua, CHI760e) with two-electrode mode.

\subsection{Contact Angle Measurements}

The water contact angle on the substrate was tested by the machine (MC-100, Changzhou Sanfeng), and it was calculated by the corresponding software. At least five tests were repeated for each sample.

\section{Results and Discussion}

\subsection{Device Design and Working Principle}

The sophisticated wearable sensor typically covered the following crucial components, referred to as "wear-sensecommunicate-analyze-interpret-decide" [27]. In our design concept, the wearable requirements must be first met, so all the electrode and sensing materials, as well as packing materials in the sensor device, are intrinsically soft and biologically compatible to cater for the wearable ability (Fig. 1a). Then the figure of merits for wearable sensors, including sensitivity, working range, or dynamic characteristics, should be carefully considered. Given that extensive reports have focused on the improvement of sensitivity, we thus primarily aimed at pushing the linear working range to a new horizon by using the soft SF as the integrated electrode for the first time in the flexible sensor (Fig. 1b), greatly expanding their applications of the flexible sensor compared to the traditional silicon-based micro-electromechanical systems (MEMS) device. Also, a promisingly large-scale way using the laser-engraved stainless steel foil as the template for casting a waterproof PDMS micropillar film (MPF) grafted with carbon nanotube (CNT) (water contact angle (CA) is approaching $150^{\circ}$ ) was employed to further improve its viability in harsh environment such as the raining (Fig. 1a). Once these abilities are thoroughly evaluated, a Bluetooth served as a wireless signal device was successfully integrated to achieve the "communicateanalyze-interpret-decide" function to send the detected signal, i.e., pulse rate or applied pressure to the portable smartphone, thereby bringing the flexible sensor from laboratory to the real world. 


\subsection{Device Design and Working Principle}

The fabricated device (Fig. 2a) mainly composed of the PDMS MPF, G-nWF, and SF electrode mounted on the PI/ PET substrate (Fig. 2b). As described above, the PDMS pillar with a diameter size of $100-\mu \mathrm{m}$ grafting CNT whiskers was successfully cast from the mold using the laser-engraved method (Fig. S1), which served as the protective armor for the device (Fig. 2c). For piezoresistive sensing materials, research has pointed to the importance of considering both conductivity and rough surface to improve its sensitivity and working range significantly [17]. In the present work, a simple yet efficient "dip-coating" method was used to fabricate such G-nWF, as shown in Fig. 2d. Hierarchical microand nano-sized non-flat surface, which was mainly induced by the texture of pristine nWF (Fig. S2), was beneficial to increasing the contacting area when subjected to pressure, thereby improving sensitivity and linear working range. Previously, typical electrode materials in sensors primarily focused on the gold, carbon, or silver-based materials fabricated using traditional lithography, screen printing as well as 3D printing method. However, few electrode structures comprising of highly uniform hierarchical architectures were explored to improve its performance further. In this work, a hierarchical was rationally employed (Figs. 2e and S5), which is directly derived from the soft composite SF using a laser-engraved method (Figs. 2f-g and S3). To gain more insight of the SF change under the illumination of the laser beam, detailed SF morphology features are shown in Figs. S5 and S6. It can be obviously observe that the SF was etched relatively smooth without any clear mechanical deformation such as curling by heating or destructive fracture. The as-obtained SF electrode exhibited a superior conductivity of $0.05 \Omega \mathrm{cm}^{-2}$ while retaining its original performance
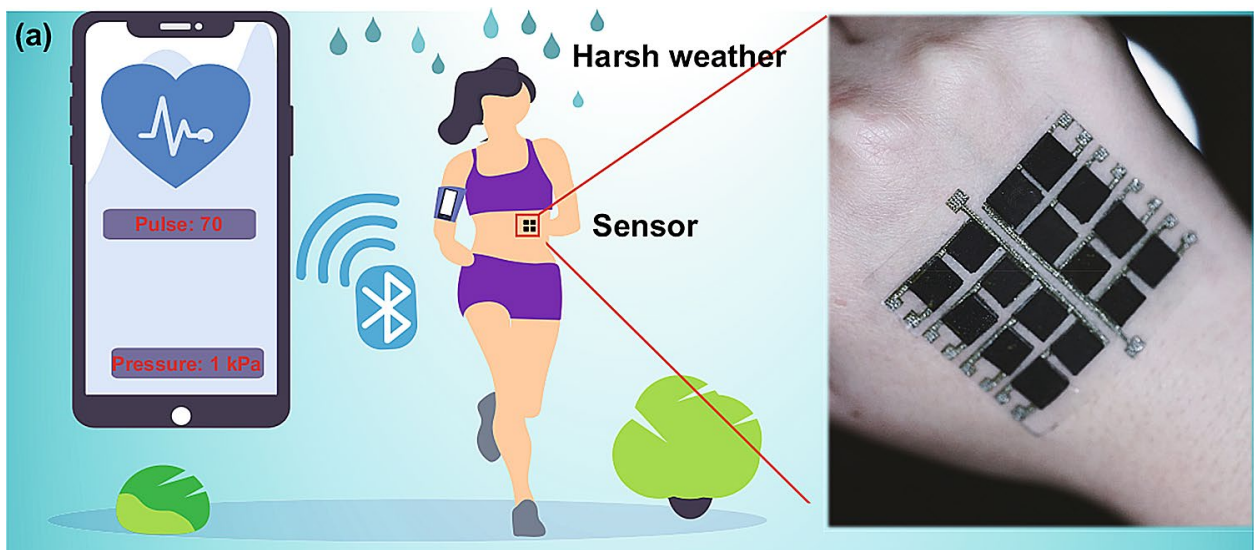

(b)

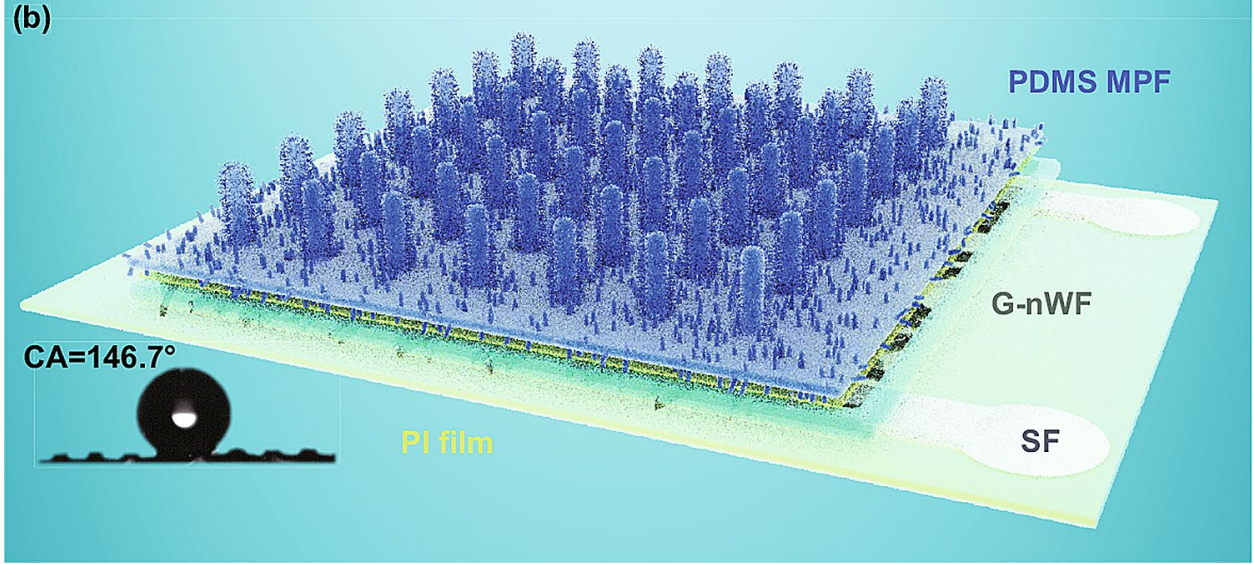

Fig. 1 Schematic illustration of the flexible sensor. a Wearable sensor capable of successfully monitoring the pulse rate and sending the signal to the smartphone via the Bluetooth and the digital optical images of the waterproof sensor mounted onto our hand. b Schematic illustration of the structure of the sensor and its waterproof properties 
(a)

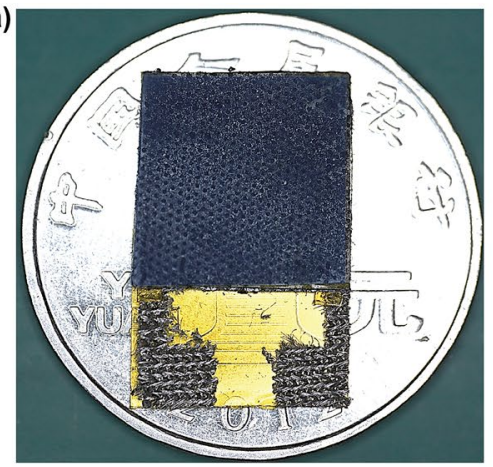

(d)

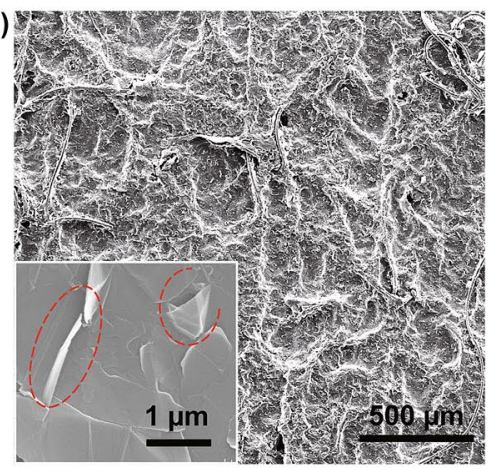

(b)

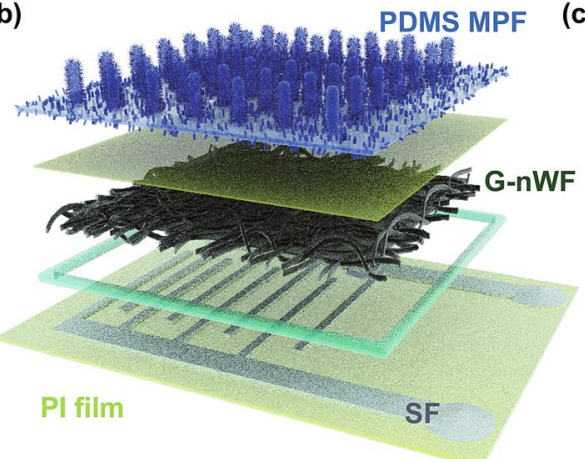

(c)

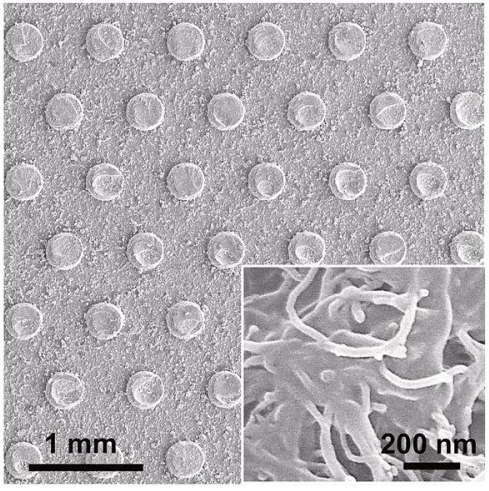

(e)
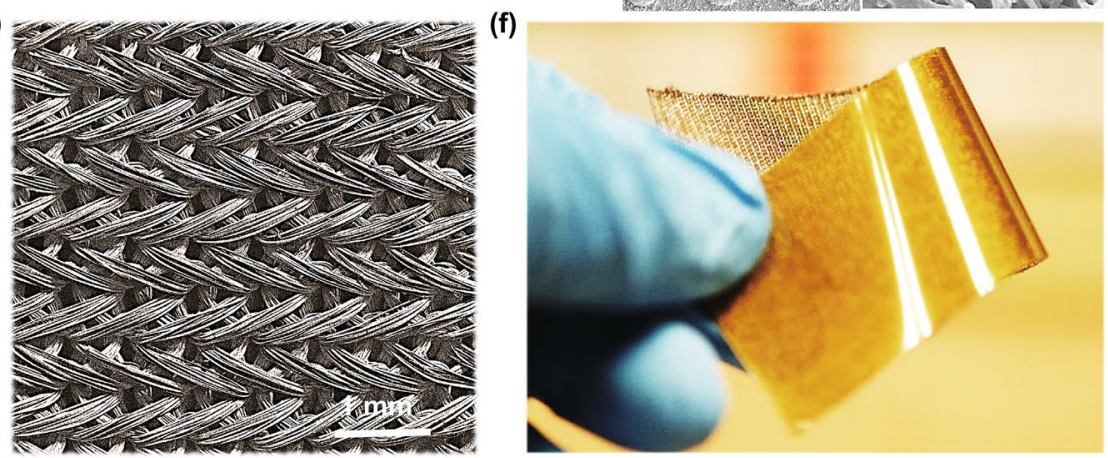

(h)

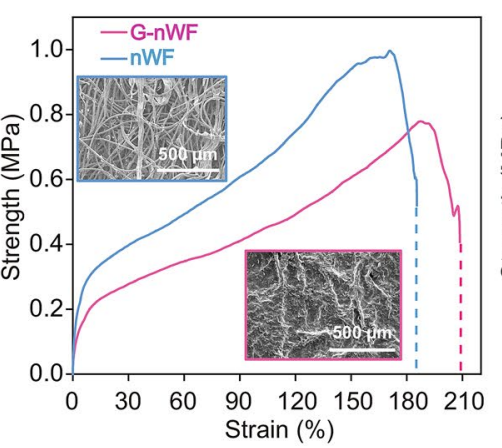

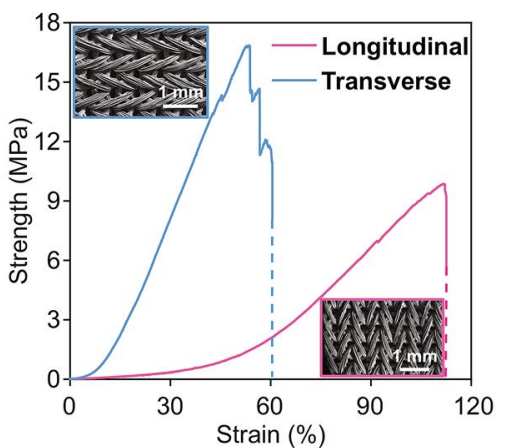

(g)

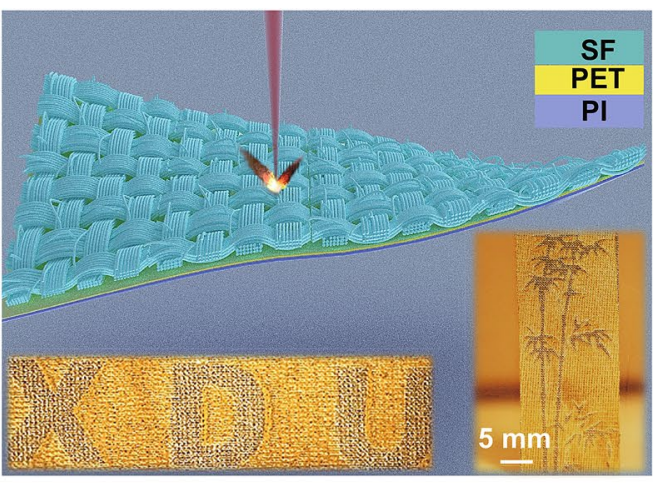




\subsection{Device Characterization}

Pressure sensors should be typically evaluated on the following items, i.e., sensitivity, working range, hysteresis, response time, and stability [17]. The sensitivity of the sensor is a critical parameter for evaluating device performance. It is typically defined as $S=\left(\Delta I / I_{0}\right) / \Delta P$, where $\Delta I$ represents the current change before and after applying pressure, $I_{0}$ represents the initial current when no pressure is applied, $\Delta P$ is the amount of pressure change from $I_{0}$ to $I$. As shown in Figs. $3 \mathrm{a}$ and $\mathrm{S} 10$, the $\Delta I / I_{0}$ as a function of pressure is plotted. The results show a superior sensitivity of $6.417 \mathrm{kPa}^{-1}$ and desired linear relation between the current change and pressure from 1.2 to 1.4 MPa, achieving an excellent balance between the sensitivity and working range. The relative stable linearity of the sensor's current variation over $800 \mathrm{kPa}$ shows the viability in extreme press loading of our device. Even below $1.2 \mathrm{kPa}$, the sensor still showed reliable sensitivity of $1.1 \mathrm{kPa}^{-1}$ and the pressure resolution of the sensor is $\sim 14 \mathrm{~Pa}$. To our scientific knowledge, only a few reports on such a linear wide working range of the flexible sensors can be found. Under a dynamic pressure of $20 \mathrm{kPa}$, the sensor remains nearly negligible hysteresis, as shown in Fig. 3b. A pressure of $50 \mathrm{kPa}$ was applied to the sensor for $7000 \mathrm{~s}$ to test its stability. However, no obvious decay was observed during this process, demonstrating its potential in practical application (Fig. 3c). Additionally, two higher pressures of $400 \mathrm{kPa}$ and $1000 \mathrm{kPa}$ were applied for $3500 \mathrm{~s}$ and the current response of $1000 \mathrm{kPa}$ is nearly twice that of $400 \mathrm{kPa}$, exhibiting the excellent linearity as well as a fast response time of only $36 \mathrm{~ms}$ (Fig. S10). This superior performance not only derived from the mechanical stability, but also from the superior robust conductivity of the silver layer of the SF. Note that the most significant merit of our sensor is the rational combination of the extended working range and superior high sensitivity, which is higher than other reports with similar sensing materials (Fig. 3d) [28-37]. Furthermore, fast response time is imperative for the sensor to process the input data. As shown in Fig. 3e, a drop of water $(\sim 0.3 \mathrm{~mL})$ fell on the surface of the sensor to test its response time and self-cleaning performances. The water would quickly be removed by the sensor due to its hydrophobic property (CA is $146.7^{\circ}$ and roll-off angle is less than $8^{\circ}$ ) of the PDMS pillar buildings, demonstrating its application in wet conditions (Figs. 3f, S11, S12, and Movie S1). Additionally, the sensor showed an excellent response time of $\sim 4 \mathrm{~ms}$, and it even can detect the droplet bouncing on the superhydrophobic surfaces (Fig. 3g), thus offering a useful tool for the bouncing testing of the water droplet, in addition to a high-speed camera. We also investigated the sensor resistance changes versus different relative humidity from 20 to $90 \%$ inside the practical environment (Fig. S13). It can be found the sensor still can work at the highest humidity environment, demonstrating the sensor's superior viability.

To elucidate the sensing mechanism of the sensor, the corresponding illustration is depicted in Fig. 4a. The resistance is primarily consisting of the resistance change of the SF $(R s)$, the G-nWF $(R w)$, and the contact resistance $(R c)$. Initially, the SF and G-nWF would undergo mechanical deformation due to the intrinsic soft properties under the applied pressure, which possibly leads to the resistance change of the $R s, R w$, and $R c$. However, as the quantitative analysis shown in Fig. $4 \mathrm{~b}$, there is almost no noticeable change of the $R s$ and only a slight variation of $R w$ during the whole applied pressure varied from 0 to $1 \mathrm{MPa}$. This confirmed that the resistance variation is mainly derived from the contact resistance but not the intrinsic bulk resistance of SF or G-nWF. Further, considering the hierarchical and unique pattern of the electrode materials (Figs. 2e and S5), the contact resistance $R c$ is primarily induced by the increased contacting area between the G-nWF and SF as a simple illustration in Fig. S14. To gain more insight, we made a comparison of our sensor with others' work which also used the same integrated electrode and carbon-based soft sensing materials to find the deep sensing mechanism. Firstly, Chen et al. [28] and Zhan et al. [38] both used the same soft carbon sensing materials (also same with ours) yet a planar smooth integrated electrode (differ with ours) to engineer the sensor. However, the sensor only can work below $20 \mathrm{kPa}$ with a reliable sensitivity. Therefore, we deduced that the modification of the electrode materials is critical for improving the sensor's performance. This is further confirmed by Zhang et al.'s work [12]. They patterned the rough integrated electrode and also used the soft carbon sensing materials, extending the linear working range up to $200 \mathrm{kPa}$. On the basis of these results we concluded that the wide linear working range of our sensor mainly derives from the ordered structure and soft property of the electrode materials given that a nearly same sensing materials were employed in these integrated electrode-based piezoresistive sensors. Additionally, as 

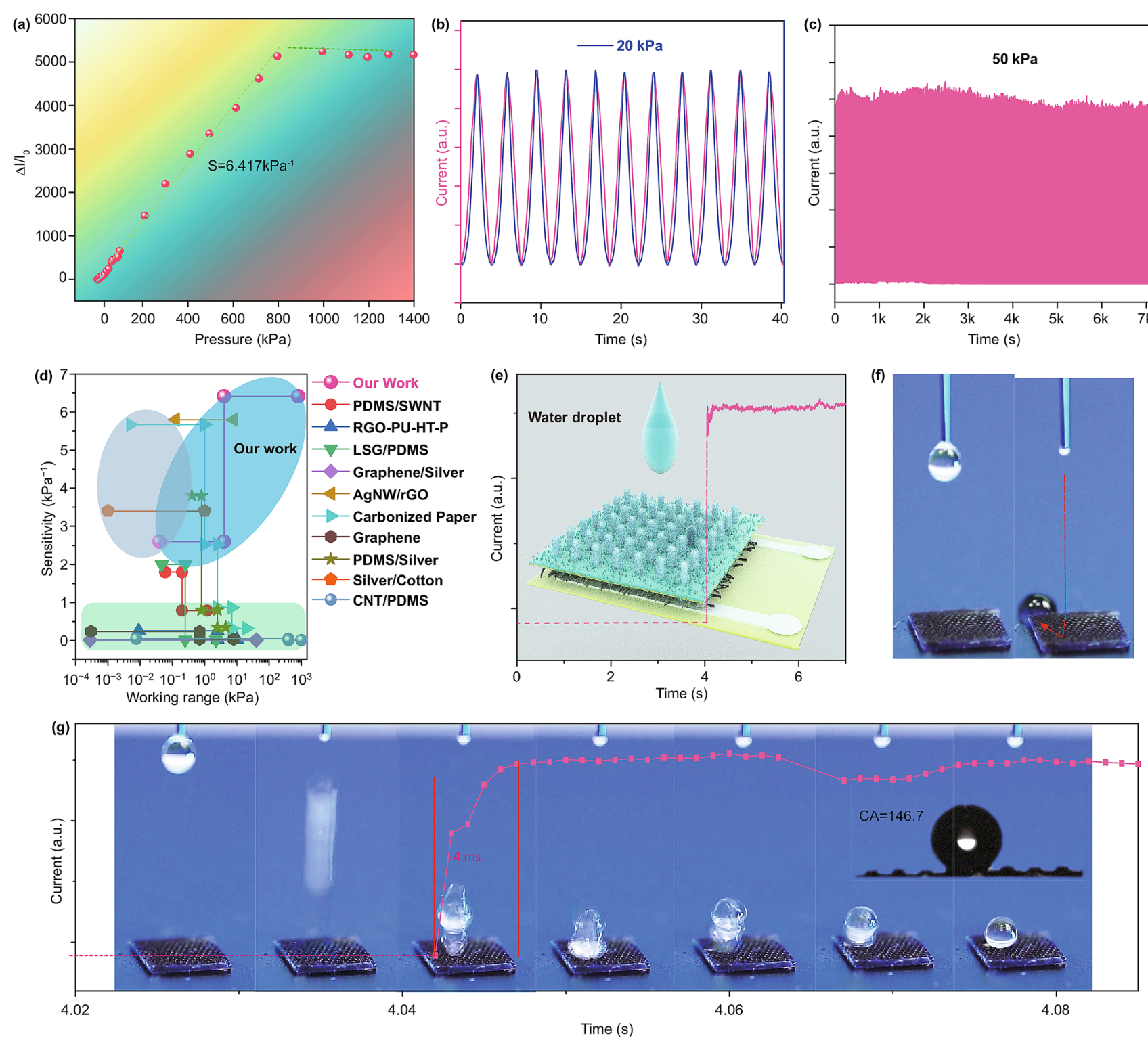

Fig. 3 The performance of the flexible sensor. a Relative current variation as a function of time for various applied stress from 0 to $1400 \mathrm{kPa}$. b The current signal has an excellent match with corresponding applied stress. c Durability test of the sensor under an applied pressure of $50 \mathrm{kPa}$ for $7000 \mathrm{~s}$. d Comparison of the sensitivity and pressure range of our sensor with the previously reported sensor. e The response of the flexible sensor when subjected to a dropping of water. $\mathbf{f}$ The water was rapidly removed by the hydrophobic surface (see Movie S1). g Our sensor can easily detect the bouncing of the water drop and showed a fast response time of only $4 \mathrm{~ms}$

the core sensing materials, the GFs' mechanical properties play an imperative role in the sensor's fast response time and long-time durability. As shown in Fig. 4c, d, the GFs are mainly consisted of 30 layers of graphene sheets (insets in Fig. 4d0 and d1). Even suffered from a serious bending ( $\mathrm{d} 0-\mathrm{d} 5)$, the GFs still can retain original state without obvious decay as demonstrated by the in situ transmission electron microscopy (TEM) nano-mechanical test. We repeated this bending process for several times, the GFs showed an excellent recoverability and flexibility (Movie S3). This also can explain why the sensor owns fast response time and superior long-time durability in addition to the excellent mechanical performance of the SF and G-nWF. 
(a)

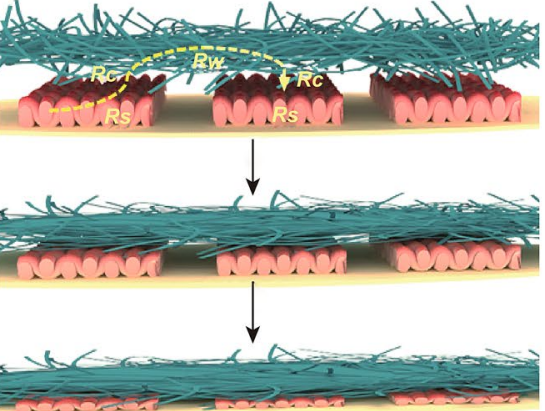

(d)

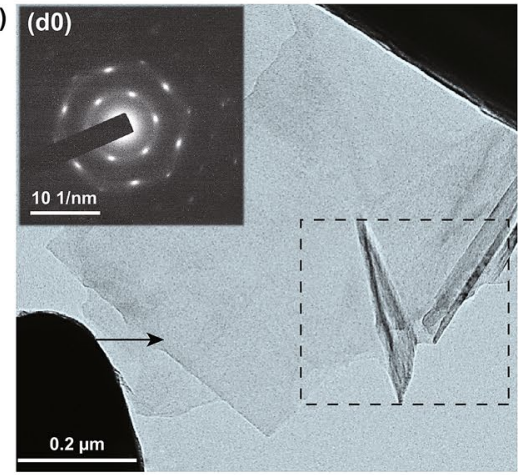

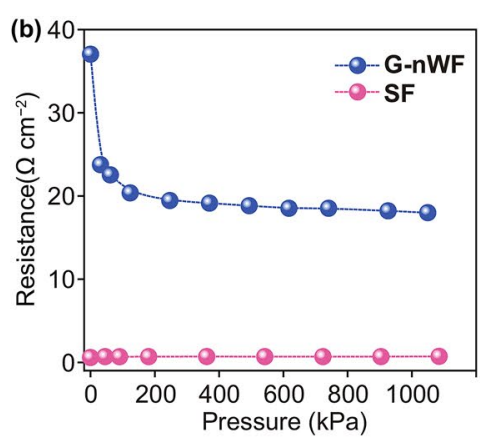

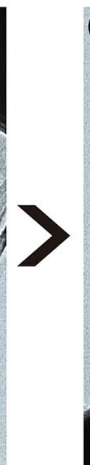

(d1)

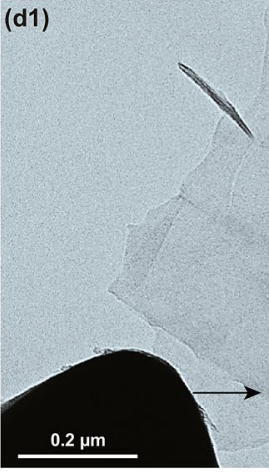

(c)

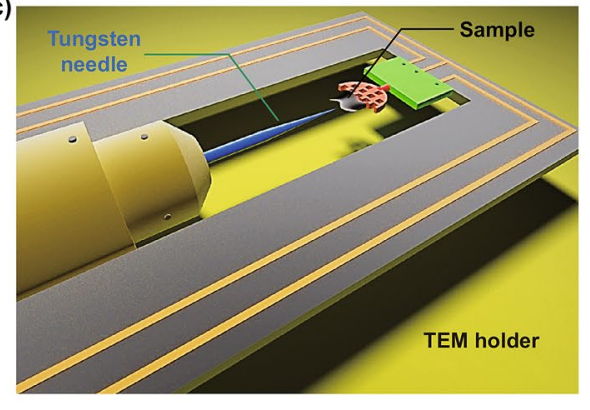

(d2)
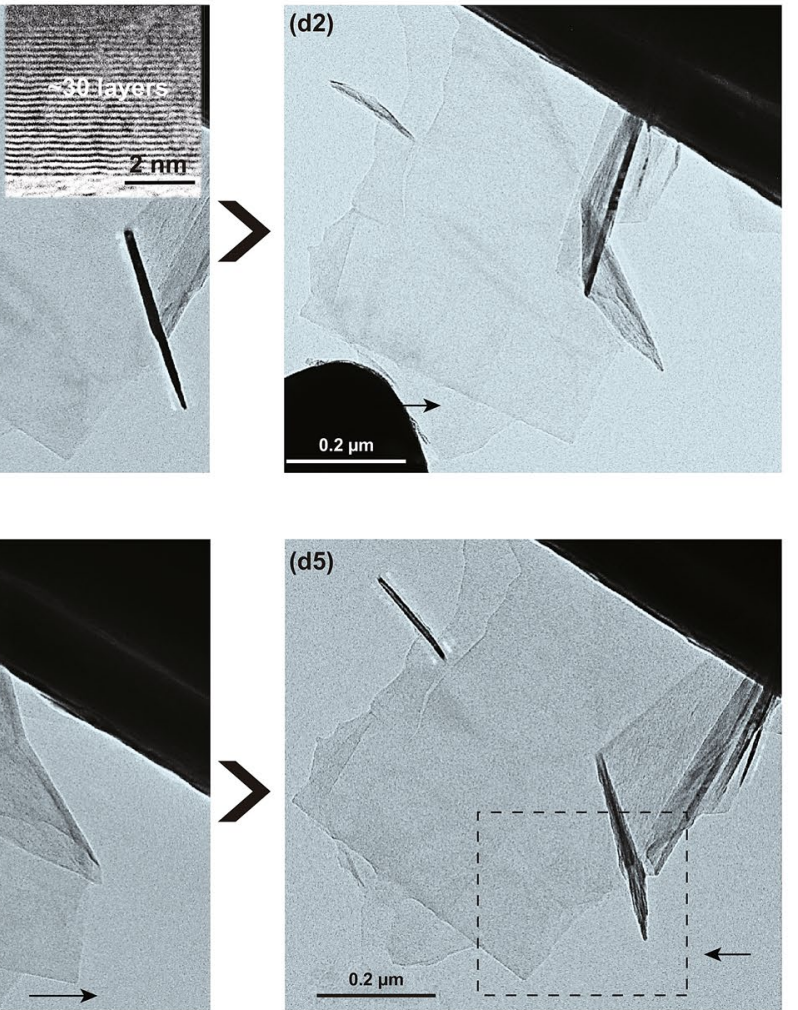
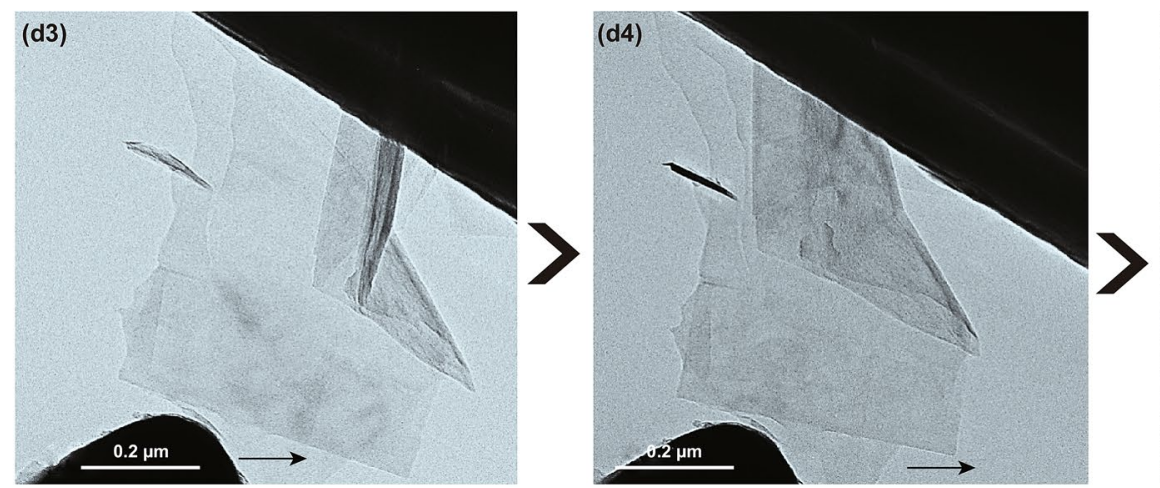

Fig. 4 Schematic illustration of the working mechanism of the sensor. a The proposed operating mechanism of the sensor. b Resistance changes of the G-nWF and SF. $\mathbf{c}$ In situ TEM micro-mechanical device for the GFs. d In situ micro-mechanical characterization of GFs inside TEM, demonstrating its superior mechanical performances

\subsection{Practical Application}

Human pulse information holds critical and potential physiological signals, which is highly related to the auxiliary diagnosis of cardiovascular disease [39]. However, the traditional rigid electronics hinders its portable and real-time detection for our better health situation. Here the flexible sensor was conformably fixed on our wrist tightly, as shown in Fig. 5a. The sensor can easily catch the pulse wave of one coauthor (27 year old, $50 \mathrm{~kg}$ ) and show the heart rate of $69 \mathrm{~min}^{-1}$
(Fig. 5b). Furthermore, the characteristic peaks of the pulse waveform, referred to Ps (percussion) wave, T (tidal) wave, and D (diastolic) wave, can be distinguished smoothly. Some signals such as the pulse velocity, time gap, and intensity ratio of the corresponding peaks related to the cardiovascular disease also can be observed, further demonstrating its practical application (Fig. 5c). The pulse waves of two different people were collected to prove its general application (Fig. 5d). Their pulse rate was calculated to be 80 and $69 \mathrm{~min}^{-1}$ for the two volunteers, respectively. Besides, 
the frequency spectrum of them was obtained through fast Fourier transformation (FFT) in Fig. 5e and f, respectively. Both the frequencies were located in the normal range for a healthy person [40]. These above results not only demonstrate the capability of the sensor to reveal the pulse information for a person but also can reflect the individual difference based on the superior sensitivity of our sensor.

Furthermore, portable commercial electronics such as the sports watch have been widely developed to make life more convenient and healthier through the real-time data collection of physiological information. However, these rigid characteristics limit the wearer's comfort and the ability to apply around the clock, so the wireless wearable and comfortable ability is therefore pursued [41]. The flexible sensor therefore was configurated with the voltage divider, low-pass filter, microcontroller, and Bluetooth to send the signal to the mobile phone or cloud computing center (Fig. 6a). As shown in Fig. $6 \mathrm{~b}$ and Movie S4, the sensor was attached to the body only by thin scotch tape, and then the pulse waves were positively observed through the customized software on the cellphone. The estimated pulse rate of $69 \mathrm{~min}^{-1}$ (inset in Fig. 6b) is close to the data obtained by the commercial smartwatch (66-68 $\mathrm{min}^{-1}$, Fig. 6c). Still, the commercial electronics present less comfort degree as it needs a very tight bonding on the body to get the true value (as marked by the white circle, Fig. 6c). Also, a continuous gentle touch was precisely revealed on the smartphone (Fig. 6d and Movie S5). Additionally, our sensor was applied to the planter pressure on different location of the subject's left foot and the maxpressure over $400 \mathrm{kPa}$ was easily detected. Hence, the plantar pressure distribution though integrating this sensor would be in favor for optimizing shoes' architecture design (Figs. S10 and S15). Based on the above information, it is strongly believed that this kind of sensor holds strong potential in integrating into flexible electronics with comfortability and portable features. Finally, building pressure sensor matrices are (a)
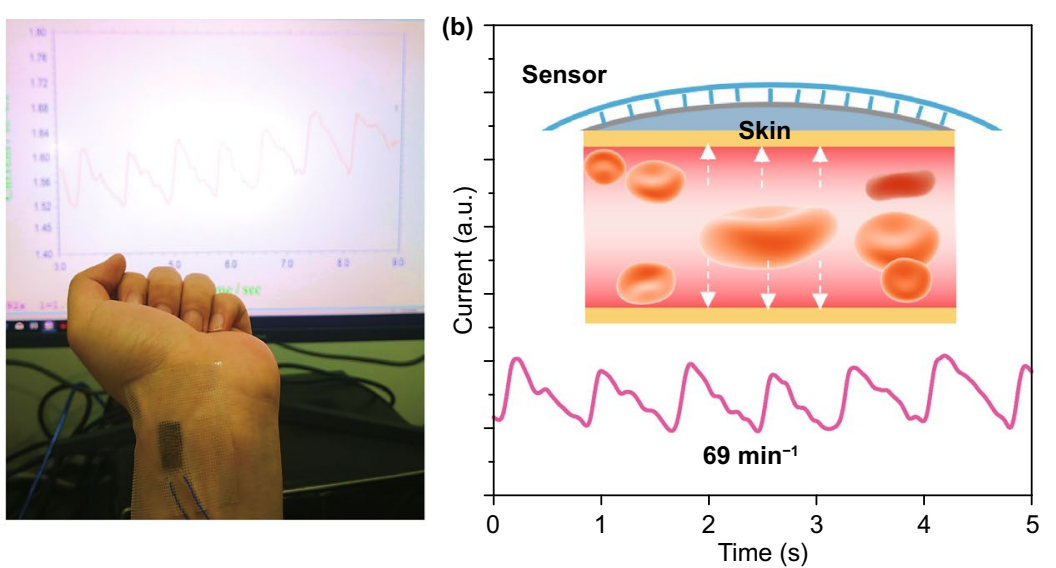

(d)

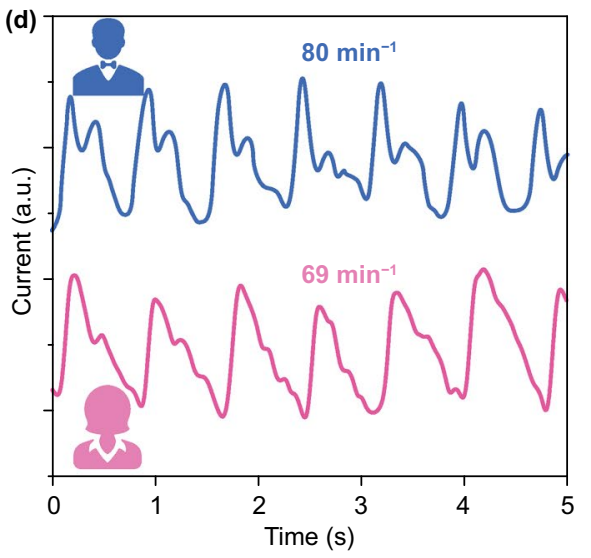

(e)

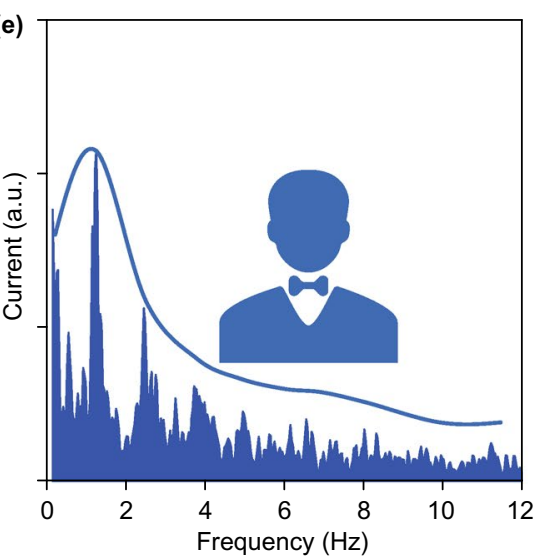

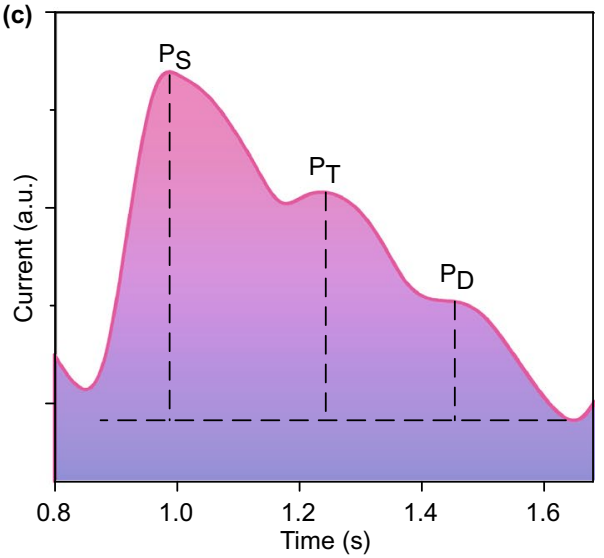

(f)

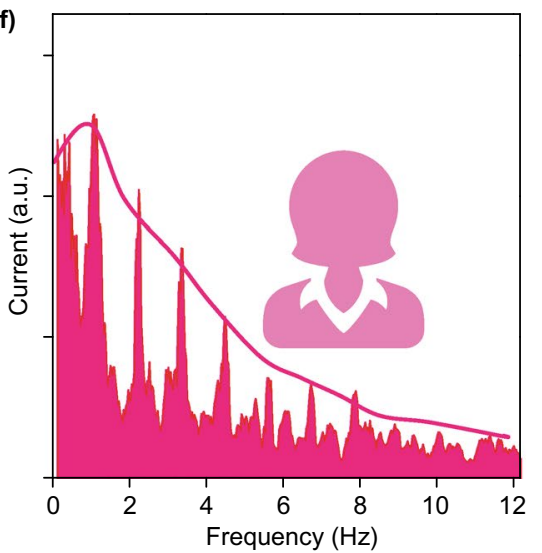

Fig. 5 Pulse rate monitoring of the flexible sensor. a The flexible sensor was well mounted onto our body to monitor the pulse rate. b-c Principle and practical application of the sensor on successfully detecting the pulse rate. d-f The sensor was able to identify different person's pulse rate 
necessary to achieve various applications on AI, touch screen panels, and robotic imaging systems [21]. Figure 6e shows a $4 \times 4$ sensor array and its corresponding highly flexible circuit diagram. The panel can show the pressing geometrical area of the weight through the current intensity, further indicating its large-scale capability for practical application (Fig. 6f). (a)
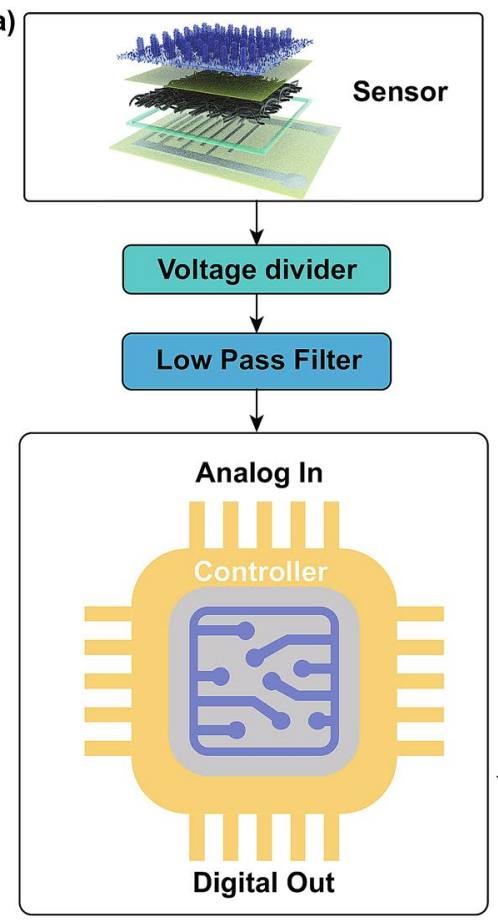

(c)

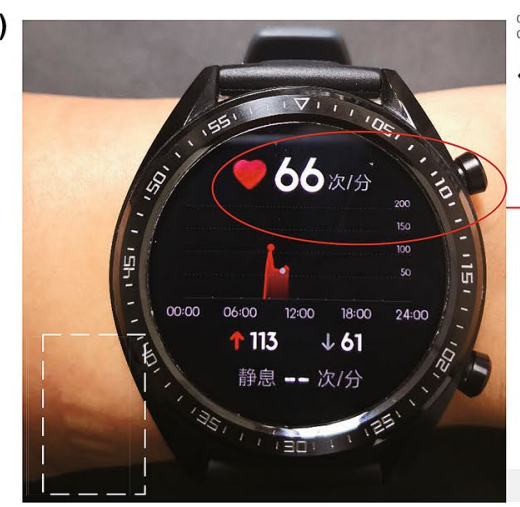

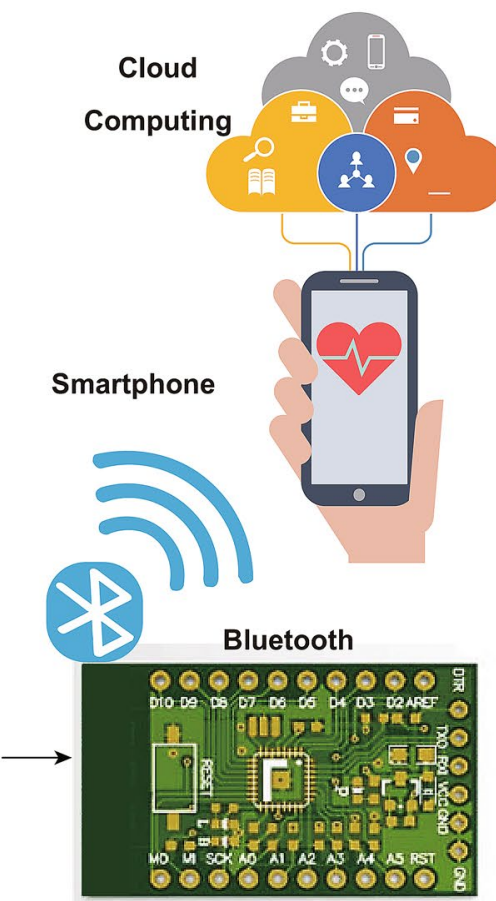

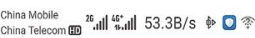

$\leftarrow$ Heart rate $v$ (e)

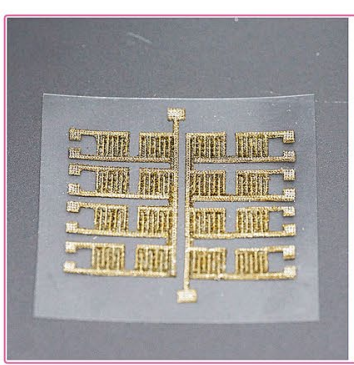

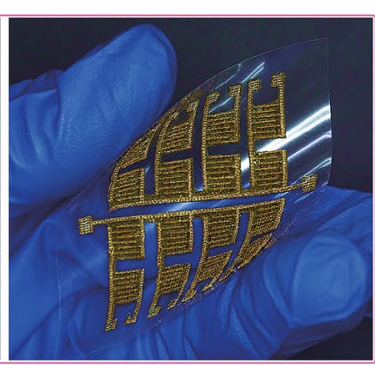

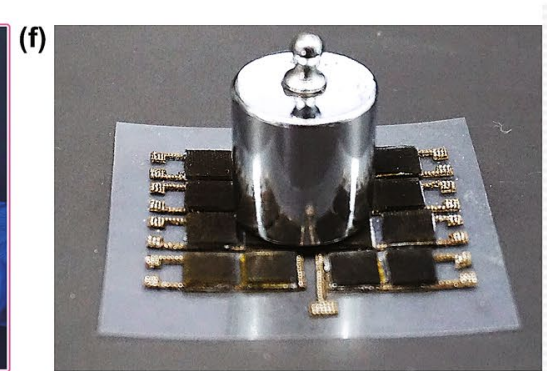

(b)

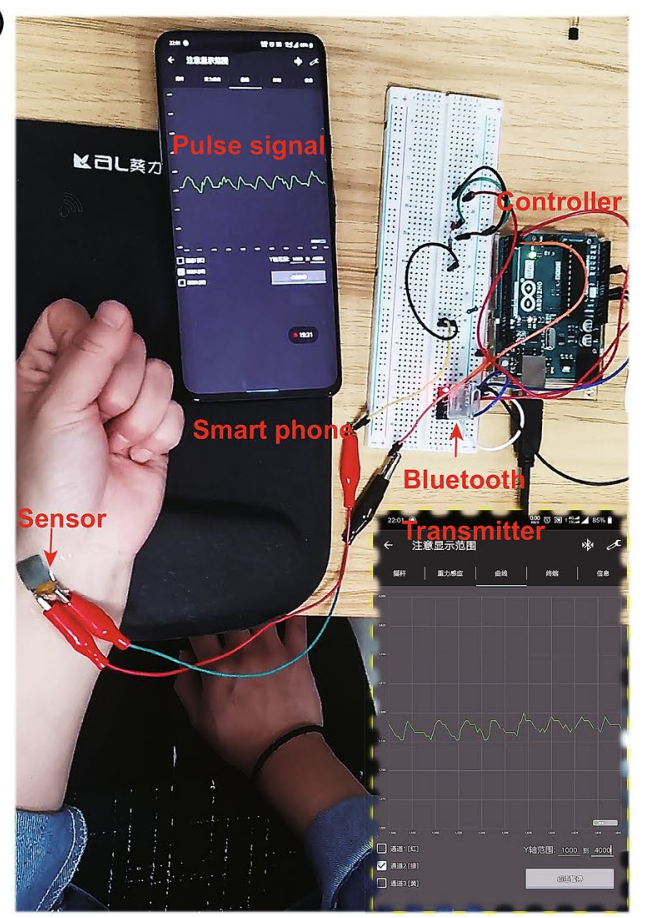

(d)

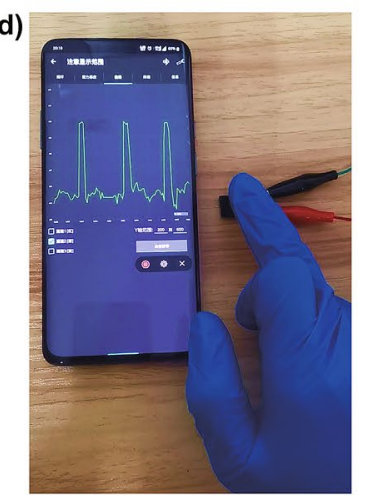

Fig. 6 Wireless real-time pulse monitoring of the sensor for healthcare. a Device configuration of the wearable sensor system. b Practical application of detecting the pulse wave and successfully send the signal to the smartphone. c Commercial sports watch showed a similar date value collected by our wearable sensor. d Practical application of gentle touching. e $4 \times 4$ sensor array and its corresponding highly flexible circuit diagram. f Pressing geometrical area of the weight measured by the sensor array 


\section{Conclusions}

In summary, we designed an effective and general approach to fabricate a soft electrode for the high-performance flexible sensor. The fully soft SF materials as the integrated electrode push the sensor's linear working range to a new level (up to $800 \mathrm{kPa}$ ) while remaining an admirable sensitivity $\left(6.417 \mathrm{kPa}^{-1}\right)$ and long-time durability without any obvious decay. Moreover, the sensor showed an ultrafast response time of only $4 \mathrm{~ms}$ based on the unique and ordered structure of the soft electrode and the sensing materials. Meanwhile, the waterproof PDMS MPF grafted with CNT anchored on the surface acted as a protective layer of the sensor to further improve its viability in harsh environments such as the raining (CA is approaching $150^{\circ}$ ), which can also be extended to other flexible electronics. Application of the sensor or sensor matrices configured with the Bluetooth successfully achieved the "communicate-analyze-interpretdecide" function to send the detected wireless signal of pulse rate or applied pressure to the portable smartphone, thereby demonstrating its feasibility and effectiveness as a reliable electronic for health monitoring. This study confirmed that rationally building the electrode was also capable of greatly improving the sensor's performance instead of merely focusing on the sensing materials. We hoped that this design concept and sensor system could potentially pave the way for more advanced wearable electronics in the future.

Acknowledgements These authors would like to acknowledge the financial support of the project from the National Natural Science Foundation of China (No. 61904141), the funding of Natural Science Foundation of Shaanxi Province (No. 2020JQ-295), the Key Research and Development Program of Shaanxi (Program No. 2020GY-252), National Key Laboratory of Science and Technology on Vacuum Technology and Physics (HTKJ2019KL510007), and City University of Hong Kong (Project Nos. 7005070 and 9667153), as well as Shenzhen Science and Technology Innovation Committee under the Grant JCYJ20170818103206501.

Author Contributions HX, LG, and YW contributed equally to this work. LG, WW, and YL lead this project. HX and YW designed and fabricated samples, conducted experiments, and analyzed data. KC performed the in situ TEM experiment and drafted the manuscript partially. All authors contributed to the final manuscript.

Open Access This article is licensed under a Creative Commons Attribution 4.0 International License, which permits use, sharing, adaptation, distribution and reproduction in any medium or format, as long as you give appropriate credit to the original author(s) and the source, provide a link to the Creative Commons licence, and indicate if changes were made. The images or other third party material in this article are included in the article's Creative Commons licence, unless indicated otherwise in a credit line to the material. If material is not included in the article's Creative Commons licence and your intended use is not permitted by statutory regulation or exceeds the permitted use, you will need to obtain permission directly from the copyright holder. To view a copy of this licence, visit http://creativecommons.org/licenses/by/4.0/.

Electronic supplementary material The online version of this article (https://doi.org/10.1007/s40820-020-00498-y) contains supplementary material, which is available to authorized users.

\section{References}

1. B. Lee, J.Y. Oh, H. Cho, C.W. Joo, H. Yoon et al., Ultraflexible and transparent electroluminescent skin for real-time and super-resolution imaging of pressure distribution. Nat. Commun. 11, 1-11 (2020). https://doi.org/10.1038/s41467-02014485-9

2. S. Sundaram, P. Kellnhofer, Y. Li, J.Y. Zhu, A. Torralba, W. Matusik, Learning the signatures of the human grasp using a scalable tactile glove. Nature 569, 698-702 (2019). https://doi. org/10.1038/s41586-019-1234-Z

3. Z. Liang, J. Cheng, Q. Zhao, X. Zhao, Z. Han, Y. Chen, Y. Ma, $X$. Feng, High-performance flexible tactile sensor enabling intelligent haptic perception for a soft prosthetic hand. Adv. Mater. Technol. 4, 1900317 (2019). https://doi.org/10.1002/ admt.201900317

4. B. Liang, Z. Zhang, W. Chen, D. Lu, L. Yang et al., Direct patterning of carbon nanotube via stamp contact printing process for stretchable and sensitive sensing devices. Nano-Micro Lett. 11, 92 (2019). https://doi.org/10.1007/s40820-019-0323-8

5. R.M. Torrente-Rodríguez, J. Tu, Y. Yang, J. Min, M. Wang et al., Investigation of cortisol dynamics in human sweat using a graphene-based wireless mhealth system. Matter 2, 921-937 (2020). https://doi.org/10.1016/j.matt.2020.01.021

6. C. Wang, X. Hou, M. Cui, J. Yu, X. Fan et al., An ultra-sensitive and wide measuring range pressure sensor with paperbased CNT film/interdigitated structure. Sci. China Mater. 63, 403-412 (2020). https://doi.org/10.1007/s40843-019-1173-3

7. Y. Yang, Y. Song, X. Bo, J. Min, O.S. Pak et al., A laserengraved wearable sensor for sensitive detection of uric acid and tyrosine in sweat. Nat. Biotechnol. 38, 217-224 (2020). https://doi.org/10.1038/s41587-019-0321-x

8. T. Ha, J. Tran, S. Liu, H. Jang, H. Jeong et al., A chest-laminated ultrathin and stretchable e-tattoo for the measurement of 
electrocardiogram, seismocardiogram, and cardiac time intervals. Adv. Sci. 6, 1900290 (2019). https://doi.org/10.1002/ advs.201900290

9. R. Shi, Z. Lou, S. Chen, G. Shen, Flexible and transparent capacitive pressure sensor with patterned microstructured composite rubber dielectric for wearable touch keyboard application. Sci. China Mater. 61, 1587-1595 (2018). https://doi. org/10.1007/s40843-018-9267-3

10. S. Chen, Z. Lou, D. Chen, Z. Chen, K. Jiang, G. Shen, Highly flexible strain sensor based on $\mathrm{ZnO}$ nanowires and $\mathrm{P}(\mathrm{VDF}-$ TrFE) fibers for wearable electronic device. Sci. China Mater. 59, 173-181 (2016). https://doi.org/10.1007/s4084 3-016-0128-8

11. Q.J. Sun, X.H. Zhao, Y. Zhou, C.C. Yeung, W. Wu et al., Fingertip-skin-inspired highly sensitive and multifunctional sensor with hierarchically structured conductive graphite/ polydimethylsiloxane foams. Adv. Funct. Mater. 29, 1808829 (2019). https://doi.org/10.1002/adfm.201808829

12. T. Zhang, Z. Li, K. Li, X. Yang, Flexible pressure sensors with wide linearity range and high sensitivity based on selective laser sintering 3D printing. Adv. Mater. Technol. 4, 1900679 (2019). https://doi.org/10.1002/admt.201900679

13. Y. Lee, J. Park, S. Cho, Y.E. Shin, H. Lee et al., Flexible ferroelectric sensors with ultrahigh pressure sensitivity and linear response over exceptionally broad pressure range. ACS Nano 12, 4045-4054 (2018). https://doi.org/10.1021/acsnano.8b018 05

14. Z. Wang, X. Guan, H. Huang, H. Wang, W. Lin, Z. Peng, Full $3 \mathrm{D}$ printing of stretchable piezoresistive sensor with hierarchical porosity and multimodulus architecture. Adv. Funct. Mater. 29, 1807569 (2019). https://doi.org/10.1002/adfm.201807569

15. Y. Li, Y.A. Samad, K. Liao, From cotton to wearable pressure sensor. J. Mater. Chem. A 3, 2181-2187 (2015). https://doi. org/10.1039/c4ta05810k

16. E. Davoodi, H. Montazerian, R. Haghniaz, A. Rashidi, S. Ahadian et al., 3D-printed ultra-robust surface-doped porous silicone sensors for wearable biomonitoring. ACS Nano 14, 1520-1532 (2020). https://doi.org/10.1021/acsnano.9b06283

17. Y. Huang, X. Fan, S.C. Chen, N. Zhao, Emerging technologies of flexible pressure sensors: materials, modeling, devices, and manufacturing. Adv. Funct. Mater. 29, 1808509 (2019). https://doi.org/10.1002/adfm.201808509

18. N. Luo, Y. Huang, J. Liu, S.C. Chen, C.P. Wong, N. Zhao, Hollow-structured graphene-silicone-composite-based piezoresistive sensors: decoupled property tuning and bending reliability. Adv. Mater. 29, 1702675 (2017). https://doi. org/10.1002/adma.201702675

19. W. Cheng, J. Wang, Z. Ma, K. Yan, Y. Wang, H. Wang, Flexible pressure sensor with high sensitivity and low hysteresis based on a hierarchically microstructured electrode. IEEE Electron Device Lett. 39, 288-291 (2018). https://doi. org/10.1109/LED.2017.2784538

20. H. Chang, S. Kim, T.H. Kang, S.W. Lee, G.T. Yang, K.Y. Lee, H. Yi, Wearable piezoresistive sensors with ultrawide pressure range and circuit compatibility based on conductive-island-bridging nanonetworks. ACS Appl. Mater.
Interfaces 11, 32291-32300 (2019). https://doi.org/10.1021/ acsami.9b10194

21. K. Wang, Z. Lou, L. Wang, L. Zhao, S. Zhao et al., Bioinspired interlocked structure-induced high deformability for twodimensional titanium carbide (MXene)/natural microcapsulebased flexible pressure sensors. ACS Nano 13, 9139-9147 (2019). https://doi.org/10.1021/acsnano.9b03454

22. C. Zhang, W. Bin Ye, K. Zhou, H.Y. Chen, J.Q. Yang et al., Bioinspired artificial sensory nerve based on nafion memristor. Adv. Funct. Mater. 29, 1808783 (2019). https://doi. org/10.1002/adfm.201808783

23. J. Shi, L. Wang, Z. Dai, L. Zhao, M. Du, H. Li, Y. Fang, Multiscale hierarchical design of a flexible piezoresistive pressure sensor with high sensitivity and wide linearity range. Small 14, 1800819 (2018). https://doi.org/10.1002/smll.201800819

24. L. Gao, R. Fan, W. Zhou, X. Hu, K. Cao, W. Wang, Y. Lu, Biomimetic and radially symmetric graphene aerogel for flexible electronics. Adv. Electron. Mater. 5, 1900353 (2019). https:// doi.org/10.1002/aelm.201900353

25. L. Gao, Y. Wang, X. Hu, W. Zhou, K. Cao, Y. Wang, W. Wang, Y. Lu, Cellular carbon-film-based flexible sensor and waterproof supercapacitors. ACS Appl. Mater. Interfaces 11, 26288-26297 (2019). https://doi.org/10.1021/acsami.9b09438

26. L. Gao, K. Cao, X. Hu, R. Xiao, B. Gan, W. Wang, Y. Lu, Nano electromechanical approach for flexible piezoresistive sensor. Appl. Mater. Today 18, 100475 (2020). https://doi. org/10.1016/j.apmt.2019.100475

27. Y. Ling, T. An, L.W. Yap, B. Zhu, S. Gong, W. Cheng, Disruptive, soft, wearable sensors. Adv. Mater. 32, 1904664 (2019). https://doi.org/10.1002/adma.201904664

28. S. Chen, Y. Song, F. Xu, Flexible and highly sensitive resistive pressure sensor based on carbonized crepe paper with corrugated structure. ACS Appl. Mater. Interfaces 10, 34646-34654 (2018). https://doi.org/10.1021/acsami.8b13535

29. X. Wu, Y. Khan, J. Ting, J. Zhu, S. Ono et al., Large-area fabrication of high-performance flexible and wearable pressure sensors. Adv. Electron. Mater. 6, 1901310 (2020). https://doi. org/10.1002/aelm.201901310

30. X. Wang, Y. Gu, Z. Xiong, Z. Cui, T. Zhang, Silk-molded flexible, ultrasensitive, and highly stable electronic skin for monitoring human physiological signals. Adv. Mater. 26, 1336-1342 (2014). https://doi.org/10.1002/adma.201304248

31. H. Bin Yao, J. Ge, C.F. Wang, X. Wang, W. Hu, Z.J. Zheng, Y. Ni, S.H. Yu, A flexible and highly pressure-sensitive graphene-polyurethane sponge based on fractured microstructure design. Adv. Mater. 25, 6692-6698 (2013). https://doi. org/10.1002/adma.201303041

32. Y. Zhu, J. Li, H. Cai, Y. Wu, H. Ding, N. Pan, X. Wang, Highly sensitive and skin-like pressure sensor based on asymmetric double-layered structures of reduced graphite oxide. Sens. Actuators B 255, 1262-1267 (2018). https://doi.org/10.1016/j. snb.2017.08.116

33. X. Dong, Y. Wei, S. Chen, Y. Lin, L. Liu, J. Li, A linear and large-range pressure sensor based on a graphene/silver nanowires nanobiocomposites network and a hierarchical structural 
sponge. Compos. Sci. Technol. 155, 108-116 (2018). https:// doi.org/10.1016/j.compscitech.2017.11.028

34. S. Chun, Y. Kim, H.S. Oh, G. Bae, W. Park, A highly sensitive pressure sensor using a double-layered graphene structure for tactile sensing. Nanoscale 7, 11652-11659 (2015). https://doi. org/10.1039/c5nr00076a

35. Y. Joo, J. Byun, N. Seong, J. Ha, H. Kim et al., Silver nanowire-embedded PDMS with a multiscale structure for a highly sensitive and robust flexible pressure sensor. Nanoscale 7, 6208-6215 (2015). https://doi.org/10.1039/c5nr00313j

36. Y. Wei, S. Chen, Y. Lin, X. Yuan, L. Liu, Silver nanowires coated on cotton for flexible pressure sensors. J. Mater. Chem. C 4, 935-943 (2016). https://doi.org/10.1039/c5tc03419a

37. Y. Wei, S. Chen, X. Dong, Y. Lin, L. Liu, Flexible piezoresistive sensors based on "dynamic bridging effect" of silver nanowires toward graphene. Carbon 113, 395-403 (2017). https://doi.org/10.1016/j.carbon.2016.11.027
38. Z. Zhan, R. Lin, V.T. Tran, J. An, Y. Wei, H. Du, T. Tran, W. $\mathrm{Lu}$, Paper/carbon nanotube-based wearable pressure sensor for physiological signal acquisition and soft robotic skin. ACS Appl. Mater. Interfaces 9, 37921-37928 (2017). https://doi. org/10.1021/acsami.7b10820

39. Y. Fu, S. Zhao, L. Wang, R. Zhu, A wearable sensor using structured silver-particle reinforced PDMS for radial arterial pulse wave monitoring. Adv. Healthc. Mater. 8, 1900633 (2019). https://doi.org/10.1002/adhm.201900633

40. T. Yang, X. Jiang, Y. Zhong, X. Zhao, S. Lin et al., A wearable and highly sensitive graphene strain sensor for precise homebased pulse wave monitoring. ACS Sens. 2, 967-974 (2017). https://doi.org/10.1021/acssensors.7b00230

41. J. He, P. Xiao, W. Lu, J. Shi, L. Zhang et al., A Universal high accuracy wearable pulse monitoring system via high sensitivity and large linearity graphene pressure sensor. Nano Energy 59, 422-433 (2019). https://doi.org/10.1016/j.nanoe n.2019.02.036 